\title{
Quantitative measurements of inorganic analytes on a digital microfluidics platform
}

\author{
Shuquan Huang ${ }^{1} \cdot$ Richard B. Fair $^{1}$ (D)
}

Received: 24 June 2019 / Accepted: 14 November 2019 / Published online: 21 November 2019

(C) The Author(s) 2019 OPEN

\begin{abstract}
Two methods were studied for selectively measuring the on-chip absorbance of trace sulfate analytes in droplets on a digital microfluidics (DMF) platform. In one method, the direction of measurement was perpendicular to the flat upper and lower surfaces of the DMF platform (vertical), and in the second method, the measurement direction was parallel to the DMF platform surfaces (horizontal). The channel height or the vertical light path length was $0.24 \mathrm{~mm}$, and the droplet diameter was $1 \mathrm{~mm}$. The DMF system employed a silicone oil transport medium whereby a thin, non-uniform oil layer formed between the droplet and the upper/lower plates which was unstable, resulting in randomly formed local oil lenses. The mobile oil lenses caused vertical absorbance measurement errors and uncertainties. The effects of the oil lenses were verified by simulation. Horizontal absorbance measurements were taken with embedded optical fibers ( $0.2 \mathrm{~mm}$ in diameter) aligned over the bottom chip surface in contact with the sides of the droplet, resulting in a horizontal light path length approximately three times that of the vertical light path. Because no oil lenses could form on the droplet's sides, the stability of repeated horizontal measurements outperformed repeated vertical measurements made on the same droplet and on multiple droplets actuated into the measurement positions. Comparisons were based on measurement standard deviations and limits of detection (LOD). The following LODs and measurement standard deviations were achieved for horizontal measurements of multiple sulfate concentrations in $1.5 \mu$ droplets: $7 \mathrm{ppm}$ for sulfate $(0.3-2.7 \%)$ and an $R^{2}$ value of 0.957 from a least square data fit. Measurements on a commercial plate reader gave comparable results ( $200 \mu \mathrm{l}$ liquid in each well, LOD equals $11 \mathrm{ppm}, \mathrm{CV}$ equals to $0.2-4 \%)$, even though the absorbance path was larger $(0.7 \mathrm{~mm})$. This LOD value means that the chip could detect $10.5 \mathrm{ng}$ of sulfate. LOD values on vertical measurements were also similar, but large measurement errors from numerous outlier points yielded an $R^{2}$ value of 0.735 and large average measurement standard deviations (36\%).
\end{abstract}

Keywords Digital microfluidics· Droplets · Sulfate measurement · Oil film instabilities

\section{Introduction}

Many mainstream chemistry, physics and biochemical analyses are based on optical detection using spectrophotometry [23] and the Lambert-Beer equation [28]. Spectrophotometry methods include the ultraviolet region, the visible region and the infrared region. A wide variety of inorganic and organic materials absorb in the UV-visible region and can be detected $[23,26,24,6]$.
Spectrophotometry is applied to chemical, metallurgical, geological, medical, food science, pharmaceutical researches and environmental monitoring systems [26, 24, $6,21,19]$. It is also widely used in water quality analysis [9]. Microfluidics is a technology aimed at miniaturizing liquid handling for developing assay systems that are smaller, faster and cheaper. By using materials such as glass, silicon and hard plastics, microfluidic devices have been demonstrated in diverse applications including organic,

Richard B. Fair, rfair@duke.edu | 'Department of Electrical and Computer Engineering, Duke University, Durham, NC 27708, USA. 
biological and inorganic molecular and particulate detection as well as molecular separations [17] enzymatic assays [1] and immunohybridization reactions. By manipulating sub-microliter liquid volumes, these devices typically use liquid flow driven by pressure or electroosmotic forces acting on continuous liquid streams or electrical and flowfocused control of droplets $[10,29]$ to control liquid routing on chip.

Digital microfluidics (DMF) is based on droplet manipulation using the electrowetting-on dielectric (EWD) effect [25]. It can be used to manipulate discrete droplets on the surface of an array electrode to complete a series of process steps and chemical reactions. The digital microfluidics system consists of top and bottom plates that sandwich a fluid layer. Today, DMF technology is often used for continuous, complex operations such as diagnostics and sample pretreatment $[12,30]$. All chemical reactions can be done on chip by droplet mixing. To measure the absorbance of inorganic analytes in a droplet without moving the droplet off of the digital microfluidics chip, an optical detection system needs to adapt to the chip structure.

A simple way to build an absorbance measuring system on a DMF chip is shown in Fig. 1. It was developed by Srinivasan et al. [27] for colorimetric enzyme-kinetic detection of glucose in droplets actuated in an oil medium. The wellmixed final droplet was moved to a specific electrode, the chromophore in the droplet absorbed the light emitted by the LED, and the amount of absorption was measured by a photodiode. In this way, with the vertical measurement path shown in Fig. 1, the distance the light travels through the droplet is only a few tenths of a millimeter. Thus, as a DMF device is scaled laterally and vertically to smaller dimensions, the vertical absorbance path length

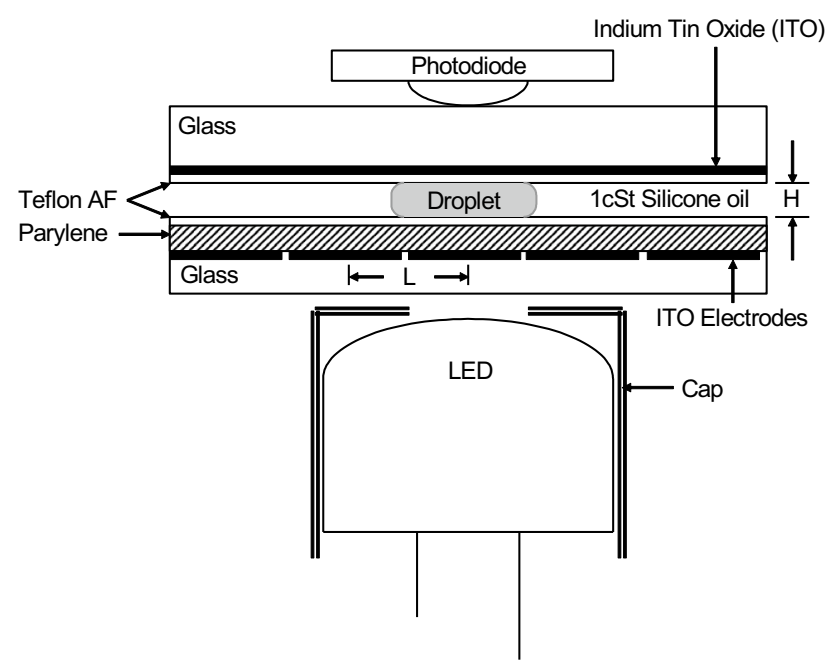

Fig. 1 The cross-sectional view of a digital microfluidics chip and the optical instrumentation for vertical absorbance measurement of dissolved glucose in a droplet [27] likewise decreases. Other work also reports on vertical path absorbance measurements [3, 31]. In another study (also a glucose concentration test) [15], the light path was made longer to increase the absorbance path length by redirecting a vertical optical path into a horizontal direction through the liquid by use of $45^{\circ}$ mirrored surfaces.

In an early study of horizontal optical waveguides on a DMF chip, a proof-of-concept system was developed to measure surface plasmon resonance of a droplet with gold nanoparticles in a saline solution system [7]. Epocore ${ }^{\mathrm{TM}}$ and Epoclad ${ }^{\mathrm{TM}}$ epoxy fibers were horizontally aligned on a DMF chip relative to an actuation electrode to build multimode optical waveguides aligned to a droplet that was actuated between the fibers. The gap between the opposing in-plane fibers could be made small enough to penetrate a droplet or the fibers could be placed outside of the droplet. Other studies have used different materials for optical waveguides, such as polydimethylsiloxane (PDMS) $[8,5]$ and SU-8 polymer [4].

Choi et al. [11] pointed out that the epoxy waveguides of Ceyssens suffered from poor transmission relative to glass optical fibers and that droplet contact with the fibers might result in sample carry-over from one measured droplet to another. Choi implemented fused silica optical fiber waveguides in the plane of a DMF device, where the opposing fibers were spaced about $4 \mathrm{~mm}$ apart and did not contact the droplets. Droplet actuation and measurements were made in an air medium. Choi reported that the curved interface of a circular droplet had a liquid lens effect that enhanced the optical signal collected by a spaced fiber by up to 2 times.

Here, a new, simple and effective way to test the absorbance in the horizontal direction using properly cleaved multimode optical fibers is presented. Unlike previous studies, the gap between the fibers or the light transmitting distance was smaller than the droplet diameter, so that droplet was squeezed between fibers. This avoids the lens effect caused by the droplet size and shape. The work also, for the first time, studied the oil lens problem encountered in measuring the absorbance in the vertical direction on a digital microfluidics chip and for the first time compared the simulated and experimental effects in measurements in both the vertical and horizontal directions under the same experimental conditions.

This paper will demonstrate the implementation of a well-developed colorimetric assay on a DMF chip, whereby limits of detection and variability of the measurements are comparable with the results obtained with a commercial plate reader (off-chip). Pre-mixed color solutions were used to generate plate reader results and initial on-chip path absorption measurements, while reagent mixing also was done on chip and the product droplet generated the on-chip results. Droplet actuation was performed 
on a DMF chip with a silicone oil medium. Two on-chip approaches were compared. The first on-chip testing approach was to build two aligned fibers in the vertical direction on the chip. This test approach proved to be incapable of stably measuring the absorbance of the droplet due to oil lenses between the droplet and the top/bottom chip surfaces. After that, the fibers were changed to the horizontal direction, whereby two in-plane fibers were sandwiched between the upper and lower plates of the DMF chip and were positioned so that the measurement droplet contacted both of the aligned fibers. Horizontal measurements were compared with vertical measurements, and the latter were found to return very consistent measurement results.

\section{Experimental}

\subsection{Chip design}

The DMF chip used in this work was based on an overall DMF chip design for performing the functions of aerosol collection of multiple inorganic particles, analyte extraction and detection in real time [14]. All functions were realized in dedicated sections on a digital microfluidics platform. Different approaches for each function were first designed and tested individually, including aerosol impaction, droplet dispensing with different kinds of reagents, droplet transport between silicon oil/air interface and across the impaction area, and colorimetric assays for sulfate, nitrate and ammonium measurement. Operation of this overall system will be presented elsewhere. In the present work, the measurement methods solely for sulfate colorimetric assays were characterized using the analyte measurement portion of the chip.

A DMF system consists of top and bottom plates that sandwich a fluid layer. The bottom plate comprises conductive electrodes that are patterned on an insulating substrate and a dielectric layer that is deposited over the electrodes. The top plate is grounded during operation. Both surfaces of top and bottom plates are modified to be hydrophobic. When a voltage is applied to an electrode, the contact angle of the droplet on the dielectric surface is changed. Electric potentials applied between the DMF electrodes and the top plate counter electrode reduce the contact angle of a droplet positioned on an electrode, thus increasing the wetting area of the droplet. Since changing the contact angle of liquid by an applied voltage can control the movement of liquid on a hydrophobic surface, a single droplet can be actuated across a dielectric covering two electrode plates with different voltages, and the droplet tends to move to the electrode with the higher applied voltage.
The bottom plate and the top plate (Fig. 2) are fabricated separately and later pressed together with a $0.24-\mathrm{mm}$-thick gasket layer $\left(0.24-\mathrm{mm}\right.$ SecureSeal ${ }^{\mathrm{TM}}$ Adhesive Sheet) put in between. The bottom substrate is on a $500-\mu \mathrm{m}$-thick borosilicate glass wafer. One-hundred and forty-nanometer indium tin oxide (ITO) is sputtered and patterned into $0.7 \mathrm{~mm} \times 0.7 \mathrm{~mm}$ electrodes with 0.05 $\mathrm{mm}$ separation space between each electrode. Then, a 1.3- $\mu \mathrm{m}$-thick parylene $\mathrm{C}$ layer is deposited as the dielectric layer. Finally, a 90-nm spin-on CYTOP (AGC Chemicals) layer covers the entire surface and the chip is baked at $100^{\circ} \mathrm{C}$ for $10 \mathrm{~min}$ followed by $190^{\circ} \mathrm{C}$ bake for $10 \mathrm{~min}$. This allowed the glass transition of CYTOP to occur and for the CYTOP to be resistant to $35 \% \mathrm{HCl}$, because in subsequent experiments, the surface of the substrate was exposed to solutions with $\mathrm{pH}=2$ and $\mathrm{pH}=12$.

The top plate was made from 1-mm-thick acrylic sheet. The top plate was completely covered with $140-\mathrm{nm}$ ITO and 90-nm CYTOP. However, the plate could not be baked, since acrylic starts melting when its temperature is above $65^{\circ} \mathrm{C}$ and top plate warping destroys the ITO conductivity. Instead, the chip is left in a desiccator overnight to allow CYTOP to dry. This makes CYTOP not resistive to acidic solutions. ITO is used to make the electrodes because it is a transparent conductive material, so the absorbance can be measured with the droplet sitting on any of the electrodes. CYTOP is used as the final hydrophobic layer because of its unique characteristics: transparency, electric insulation, water and oil repellency and chemical resistance.

The measurement chip design (Fig. 3) has 20 connection pads (long rectangular shapes) and 26 electrodes, so the chip is also a multiplexed system, meaning that one connection pad connects to multiple electrodes. This design is used for absorbance tests for inorganic ion detection, and liquids are introduced on chip into four reservoir holes drilled on its top plate. Prior to loading the reservoirs, the chip is filled with $1 \mathrm{cSt}$ silicone oil.

In almost all experiments, the bottom plate was connected to the positive voltage and the upper plate was

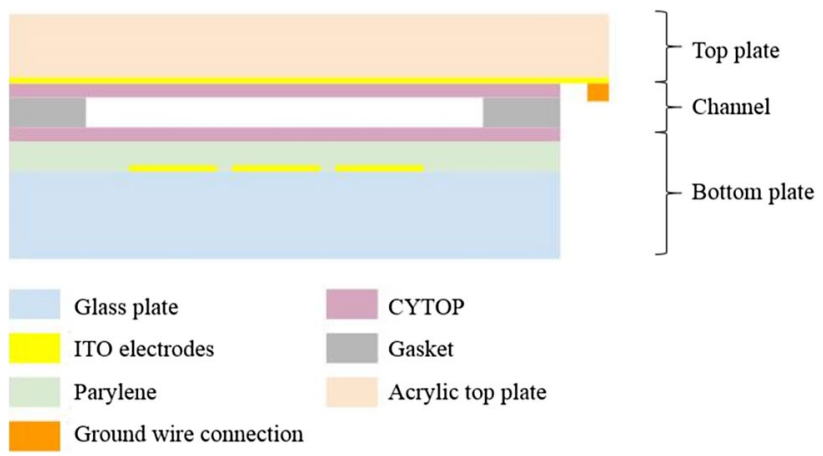

Fig. 2 The cross-sectional view of the DMF chip 


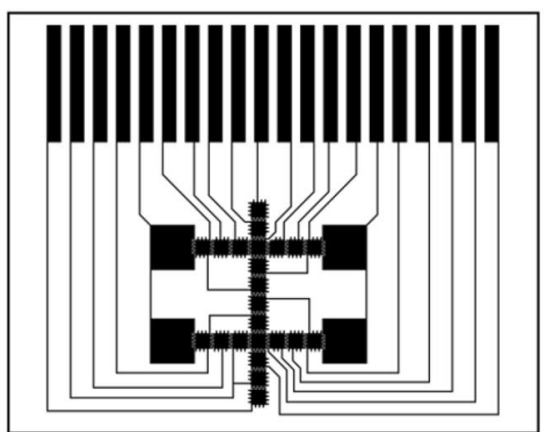

(a)

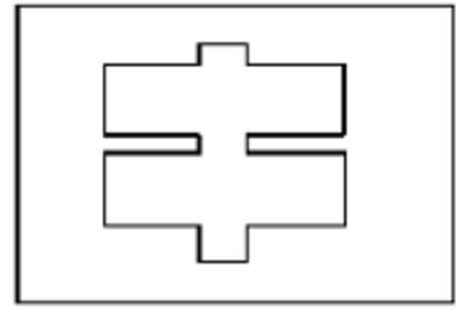

(b)

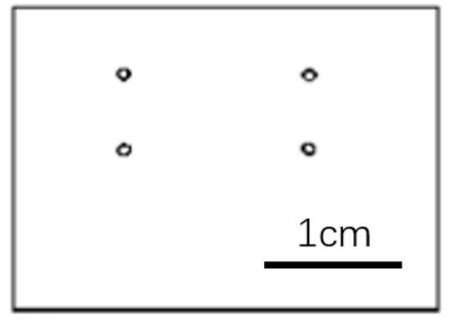

(c)

Fig. 3 Measurement chip design. a Bottom plate $(28 \times 23 \mathrm{~mm})$, b gasket, $\mathbf{c}$ top plate

grounded. The actuation voltage comes from a function generator (Agilent 33120A) and a 10 $\times$ amplifier (FLC Electronics F10A). Only in the experiment where voltage polarity was switched (Sect. 3.1.2), the connections of the top and bottom substrates were swapped every $30 \mathrm{~s}$, and the value of absorbance was compared.

\subsection{Colorimetric detection}

Methods of sulfate detection have received much attention in environmental science studies $[2,20]$. Methylthymol blue (MTB) method is a very popular method based on colorimetry. It is based on the following reactions:

$$
\begin{aligned}
& x \mathrm{SO}_{4}^{2-}+y \mathrm{Ba}^{2+} \rightarrow x \mathrm{BaSO}_{4}+(y-x) \mathrm{Ba}^{2+} \quad \mathrm{pH}=2 \\
& (y-x) \mathrm{Ba}^{2+}+z \text { MTB } \rightarrow(y-x) \text { MTB }-\mathrm{Ba} \\
& \quad+(z-y+x) \text { MTB } \quad \mathrm{pH}=12 .
\end{aligned}
$$

MTB-Ba is methylthymol blue complexed with one barium ion. Uncomplexed MTB has an absorption peak at $460 \mathrm{~nm}$, while the absorption peak of complexed MTB is at $608 \mathrm{~nm}$. However, the sensitivity of change in absorbance as a linear function of MTB-Ba at $608 \mathrm{~nm}$ is three times larger than the sensitivity change with uncomplexed MTB at $460 \mathrm{~nm}$ [18]. So, the absorbance at $608 \mathrm{~nm}$ is measured to determine sulfate concentration.

Several tests were designed to evaluate the performance of the vertical light path of on-chip absorbance measurements. Initially, pre-mixed solutions of MTB-Ba sulfate were introduced onto the chip. The absorbance of these pre-mixed solutions had already been tested with a plate reader, and the linear relationship between the absorbance and sulfate concentration had been verified. To reproduce this linear relationship on chip, it was verified that the shortened light path length [from $4 \mathrm{~mm}$ (plate reader) to $0.24 \mathrm{~mm}$ (on chip)] did not appreciably alter the signal-to-noise ratios of the measured absorbances.

The first test was to introduce each MTB-Ba sulfate premixed solution on chip and measure the absorbance. The solution was introduced by pipet into a reservoir of the chip. A droplet was dispensed and moved to the detection electrode. The droplet stayed on the detection electrode for $20 \mathrm{~s}$, moved away for $20 \mathrm{~s}$, and moved back to the detection electrode for another $20 \mathrm{~s}$, and this process was repeated several times. During the entire experiment, absorbance measurements were taken every $100 \mathrm{~ms}$ and plotted. This process was repeated for all six MTB-Ba sulfate final solutions. The spectrometer (Ocean Optics USB2000) parameters were set as follows: integration time: $3 \mathrm{~ms}$, scans to average: 5 , moving average: $5 \mathrm{~nm}$. The dark noise correction was on, and the dark spectrum was taken before turning on the LED and was automatically subtracted from later measurements.

Subsequent measurements were taken using on-chip mixed assays. The sulfate standard solutions were diluted from $120 \mathrm{ppm}$ stock solution into six different concentrations: $120,90,60,30$ and $12,6 \mathrm{ppm}$. The droplet mixing could be completed in a few seconds, and the resulting droplet transported to the on-chip detection position as soon as the mixing finished. Even if several measurements were taken to reduce random error, the measurement time took at most a few minutes. Therefore, the change in solution absorbance over time during the on-chip test could be neglected.

\subsection{Horizontal and vertical light path setup}

\subsubsection{Vertical light path setup}

In this experiment, the bottom glass plate of the chip supported indium tin oxide electrodes, so light could pass through the chip. Light was provided by a 608-nmwavelength LED and was directed by core glass clad silica 
multimode optical fiber (Thorlabs FG200UEA, $200 \mu \mathrm{m}$ core diameter) to the center of a measurement electrode on the bottom plate of the DMF device. The transmitted light was captured by another optical fiber (Thorlabs M35L01, $1000 \mu \mathrm{m}$ core diameter) from the top of the plate of the chip and was measured by spectrometer. The alignment of the two fibers was guaranteed by fixing the two fiber ends onto two machined aluminum arms. Both optical fibers had FC connectors which screwed onto the two FC adaptors at the end of the aluminum arms. The aluminum arms were aligned by two punched holes that tightly fit into two fixed vertical rods. The error from the CNC drilling and milling machine was less than $20 \mu \mathrm{m}$.

The diameter of the input optical fiber $(0.2 \mathrm{~mm})$ for the incoming light was chosen to be much smaller than the droplet diameter $(1 \mathrm{~mm})$ and the fiber head positioned at the center of the droplet, as shown in Fig. 4. This was to avoid any lens effects caused by non-uniform solutions over the diameter of the droplet. In this case, most of the light that came out of the optical fiber head passed through the droplet, even though it was not strictly collimated. However, the smaller the optical fiber diameter, the harder it was to align the input and collection fibers. Thus, based on experimentation a $0.2-\mathrm{mm}$ core diameter was selected. The digital microfluidics chip was held by a card edge connector and was mounted on a precision positioning stage. Figure 4 shows the relative sizes of the input fiber and the droplet.

\subsubsection{Horizontal light path setup}

Chips utilizing a horizontal light path were fabricated by fixing two bare optical fibers inside the chip between the top and bottom plates. To keep the optical fibers in place, a fabrication step was added before parylene deposition to build an SU-8 channel of $\sim 150 \mu \mathrm{m}$ in depth, as shown in Fig. 5. Both optical fibers used had $200 \mu \mathrm{m}$ core and $220 \mu \mathrm{m}$ cladding diameters, which was slightly less than the channel height $(0.24 \mathrm{~mm})$. The optical fibers were carefully cleaved to create a flat end surface and were coated with CYTOP, so that the droplets in the silicone oil medium could easily pass between the fibers without being pinned and without carry-over contamination. During chip assembly, the optical fibers were placed in these pre-made channels, as shown in Fig. 6. The fibers were coupled with the

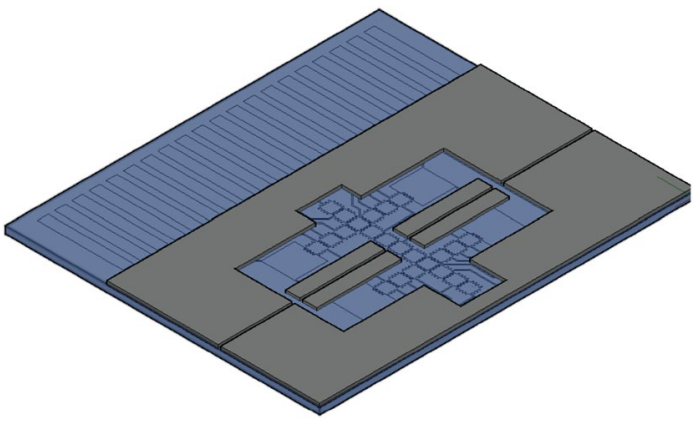

Fig. 5 A channel from SU8 to fix the optical fibers in place

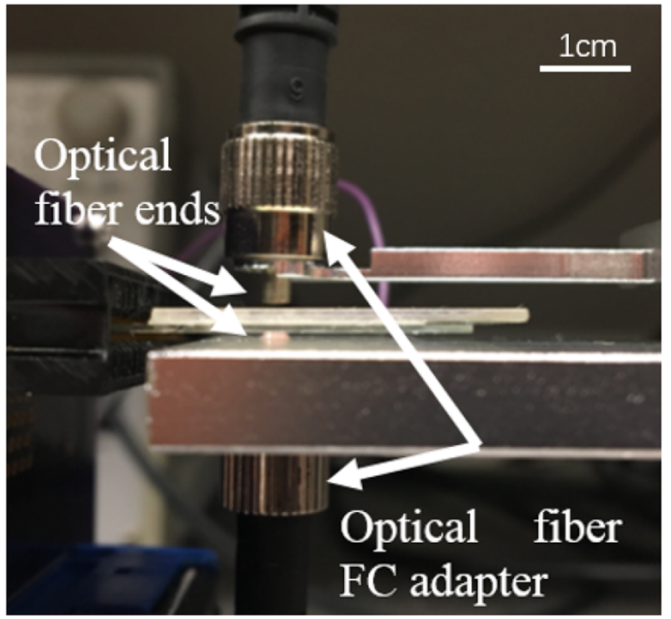

(b)
Fig. 4 a The system setup of the vertical measurement experiment. The box on the left is connected to an XYZ axis station, and the droplet is placed in the middle of the light path by fine-tuning the chip position. A PCB card edge slot connector is mounted on the motherboard in the vertical direction, and the connector pads of the chip are correspondingly inserted into the card slot, and through the motherboard and a connected ribbon cable (not shown). They are connected to the controller PCB. The two arms on the right side fix the optical fibers, and the two vertical rods ensure that the two optical fibers are vertically aligned. $\mathbf{b}$ A side view of the relative position of the vertical optical fibers and the chip 
Fig. 6 Horizontal optical fibers setup. On the right, it can be see that the fibers were placed such that a $2 \times$ droplet would actuate between the fibers to a position between two electrodes
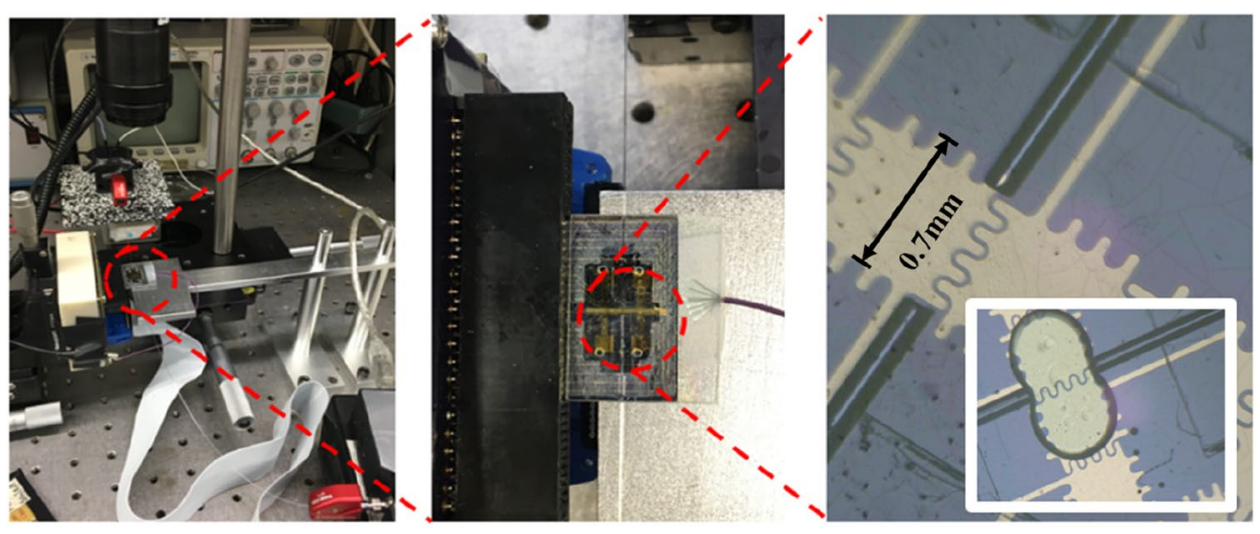

LED holder and the spectrometer using a Thorlabs BTF1

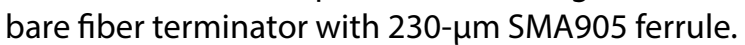

\section{Results}

\subsection{Vertical light path measurements}

The first test was to introduce each MTB-Ba sulfate premixed solution on chip and measure the absorbance. Six different concentrations of standards were chosen for calibration as mentioned in Sect. 2.2. The solution was loaded by pipette into a reservoir on the chip. A droplet was dispensed from the reservoir and moved to the detection electrode where it remained $20 \mathrm{~s}$, moved away for $20 \mathrm{~s}$, and moved back to the detection electrode for another $20 \mathrm{~s}$, and this process was repeated several times. During the entire experiment, absorbance measurements were taken every $100 \mathrm{~ms}$, and the absorbance at $608 \mathrm{~nm}$ was extracted and plotted. This process was repeated for all six MTB-Ba sulfate final solutions.

The reference bright spectrum was taken each time before introducing the MTB-Ba sulfate solution, which was the light intensity received by the spectrometer with only silicone oil inside the channel. This reference spectrum was kept same during the droplet movements and was used to calculate the absorbance every time a droplet moved to the detection position. When a droplet moved away, the absorbance would return to zero. The results show that when the droplet was away from the detection electrode, the absorbance always read in the range $0 \pm 0.003$, and this zero reading did not increase over time. This means the LED brightness was stable, the setup was stable, and the droplet did not leave any detectable carry-over residue while moving away.

Figure 7 shows the time versus absorbance measurements for solution \# 6 at 6 ppm sulfate concentration. Some spikes can be clearly seen on the absorbance-time plot, and these spikes indicate the moment when the droplet was moving onto or out of the measurement electrode, and the edge catches the light. These spikes are very helpful to distinguish whether the droplet is in position or not, especially for lower concentration solutions, when the absorbance was very close to zero.

The absorbance measurements of three other sulfate concentrations ( $60 \mathrm{ppm}, 30 \mathrm{ppm}$ and $12 \mathrm{ppm}$ ) are plotted in Fig. 8 over time with a vertical optical detection path on chip. The results show several interesting things:

1. During each of the 20-s measurement periods, the absorbance was stable, except for one measurement with solution \#4 (sulfate concentration $=30 \mathrm{ppm}$ ).

2. The absorbance measured in different periods can be very different. For example, the absorbance measured with solution \#3 shown in Fig. 8 in the period around $400 \mathrm{~s}$ was more than $100 \%$ larger than the absorbance measured in other periods.

To evaluate the absorbance changes from different periods, the average of 200 data points in each period was taken, representing the absorbance level in that specific period. Thus, each solution had 20 data points of this averaged absorbance. These points are plotted in Fig. 9. The solutions with higher sulfate concentration (lower absorbance) were more affected by the variance between measurements. Solution 1 (120 ppm) had the lowest average absorbance, and its coefficient of variance was $95 \%$.

In Fig. 9, linear regression was done with all 120 data points, and the $R^{2}$ value was 0.7348 , indicating that the absorbance obtained from on-chip measurements and the sulfate concentration was somewhat correlated, but the error could be large when measuring sulfate solutions with unknown concentrations. From the graph, $12 \mathrm{ppm}$ and $60 \mathrm{ppm}$ had more consistent absorbance measurements, but $60 \mathrm{ppm}$ also had outlier points. Outlier points were common during several repeated tests. 6 ppm, 30 ppm $90 \mathrm{ppm}$ and $120 \mathrm{ppm}$ showed a wide range of measurements, indicating that each reading was subject to large 
MTB-Ba sulfate final solution \#6 6 ppm
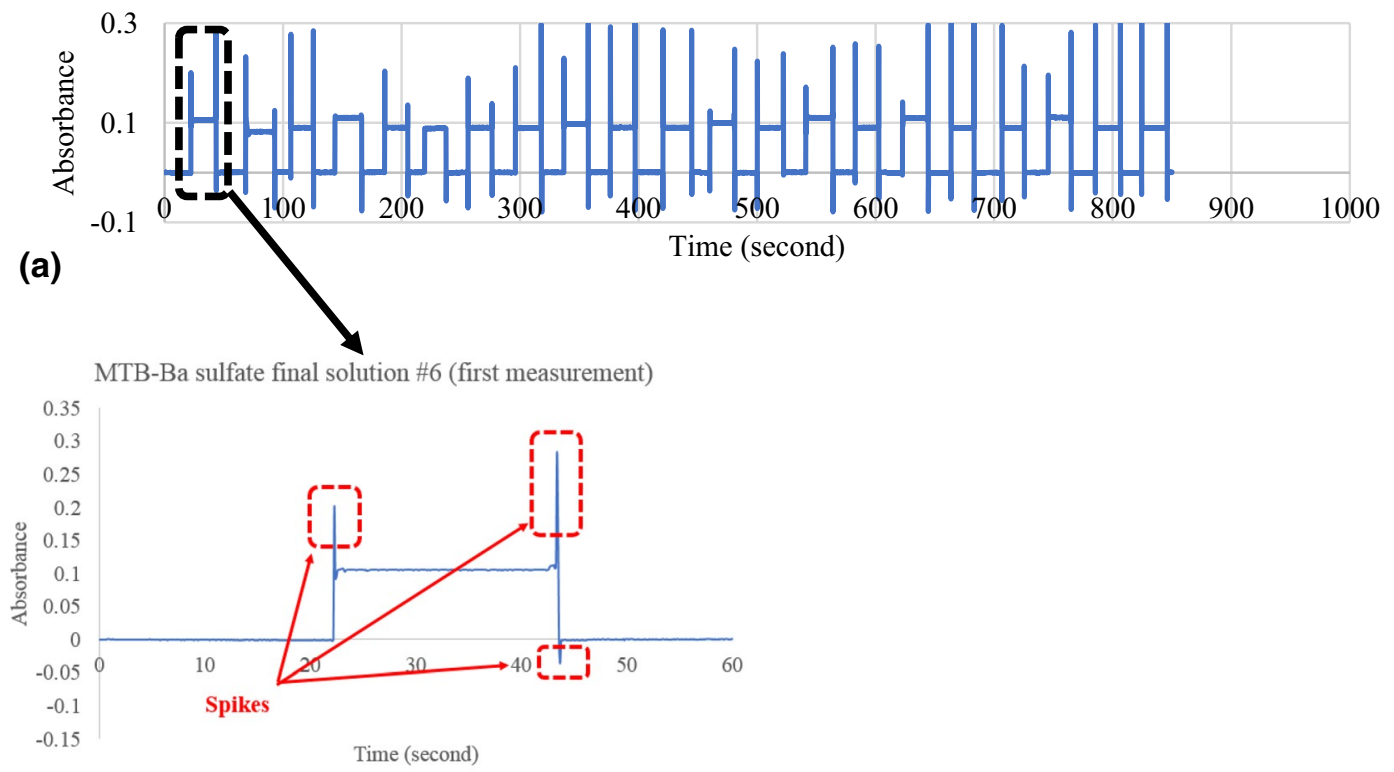

(b)

Fig. 7 The absorbance measurements of sulfate solution \#6 from Sect. 2.2 over time with a vertical optical detection path on chip. b magnifies the first measurement of solution 6 , and it is clear that the edge of the droplet caused the absorbance to suddenly rise as the droplet entered and left the range of the light path
Fig. 8 The absorbance measurements of three other sulfate concentrations ( $60 \mathrm{ppm}$, $30 \mathrm{ppm}$ and $12 \mathrm{ppm}$ ) over time with a vertical optical detection path on chip

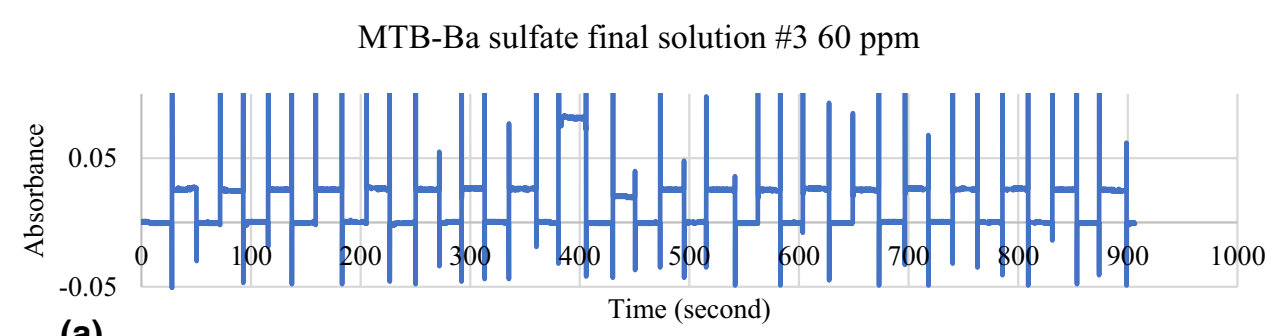

(a)

MTB-Ba sulfate final solution \#4 30 ppm

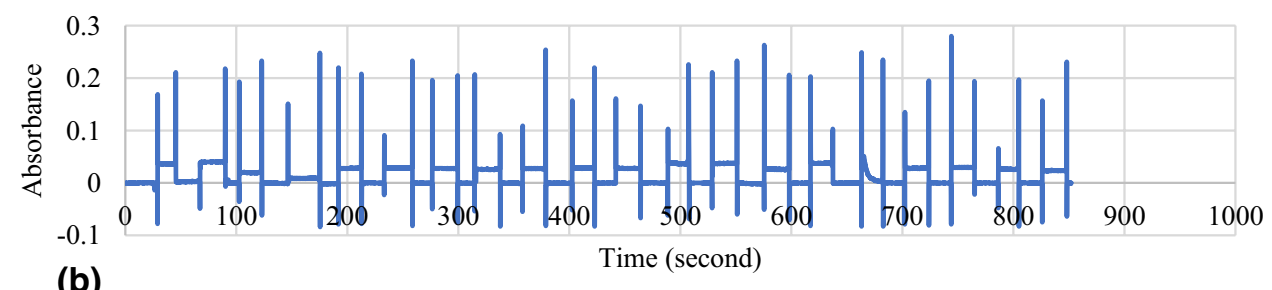

(b)

MTB-Ba sulfate final solution \#5 12 ppm

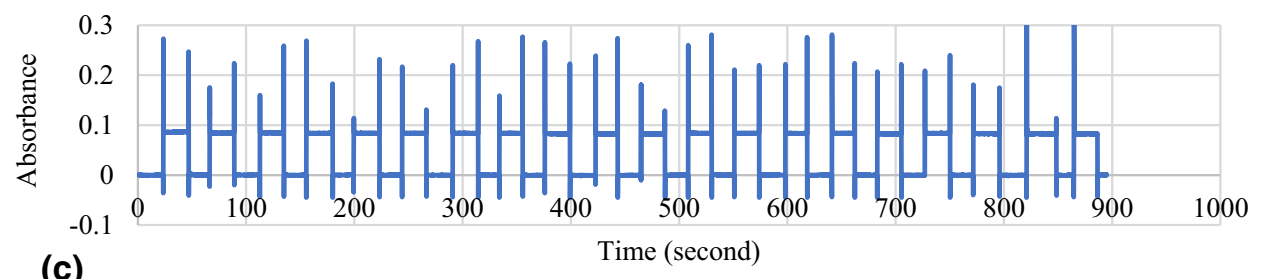

(c) 


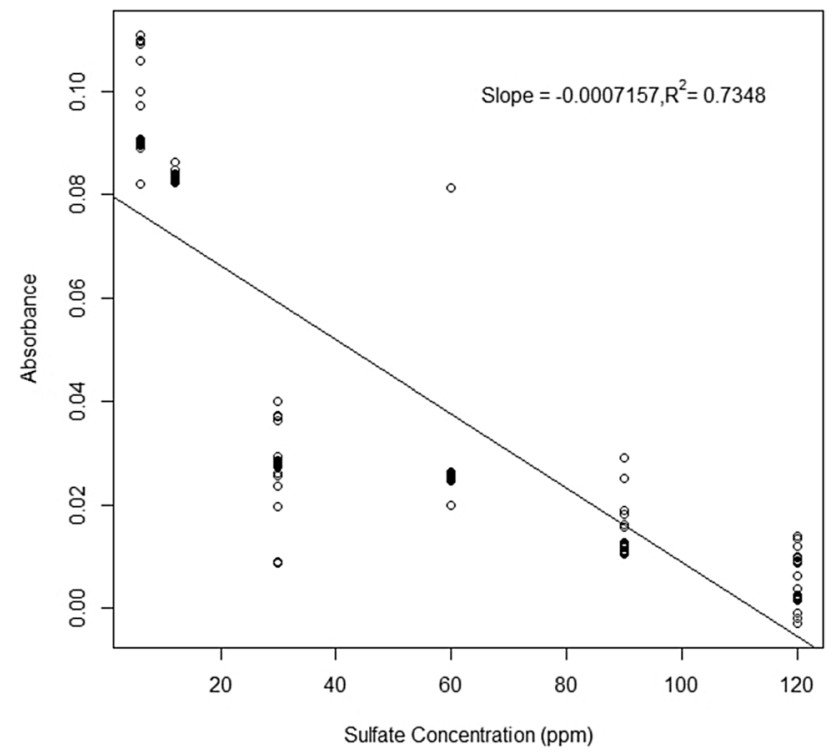

Fig. 9 The 20 absorbance measurements (averaged over the measuring period) for each of the six sulfate concentrations

interference, and a random reading could deviate significantly from the regression line.

\subsubsection{Droplet moving from different directions with vertical light path}

Since the whole experimental setup including the chip was not the cause for the variation in readings and the variation seemed to occur randomly with the movements of the droplet, it would be interesting to try moving the droplet in different directions and find out if this would cause the readings to be different. A DI water droplet with blue food dye was used in this test. Figure 10 shows one droplet being moved to the detection electrode and away for three times from different directions.

It can be seen in Fig. 10 that small round thin island films of oil are observed in the images. These island films are between the droplet surface and the upper and lower plates in an electrowetting device $[13,16,22]$. In these oil layers, so-called oil lenses can form due to the presence of capillary waves in the oil film during the movement of the droplet. When the droplet stops moving by actuation, the local oil film thickness is uneven. This happens near both the upper and lower plates. A recent study showed that the morphology and duration of the oil lenses are related to the activation voltage, actuation frequency and the use of a surfactant, and at some critical conditions, the oil film is broken [16]. Here, the oil lenses appeared frequently and did not disappear with time.

The present work experimentally found that when a droplet stopped moving and stayed on an electrode, the position and size of the oil lenses were also fixed. However, the size and position of the oil lenses changed after the drop moved away from and back to its original position (Fig. 11). Arrows point to some of the larger oil lenses. It is not clear whether these lenses occurred on the bottom plate or the top plate, because the depth of field of the optical microscope was larger than the thickness of the channel, and interference microscopy should be used to determine the accurate height of these lenses. Figure 11 [16] shows the formation of oil lenses at the oil film under the top plate. The oil lenses of the upper and lower plates are basically indistinguishable. In both positions, large diameter lenses and small and dense lenses can be formed.
Fig. 10 The oil lens position and size change after three movements. The arrows are pointing to some visible oil lenses

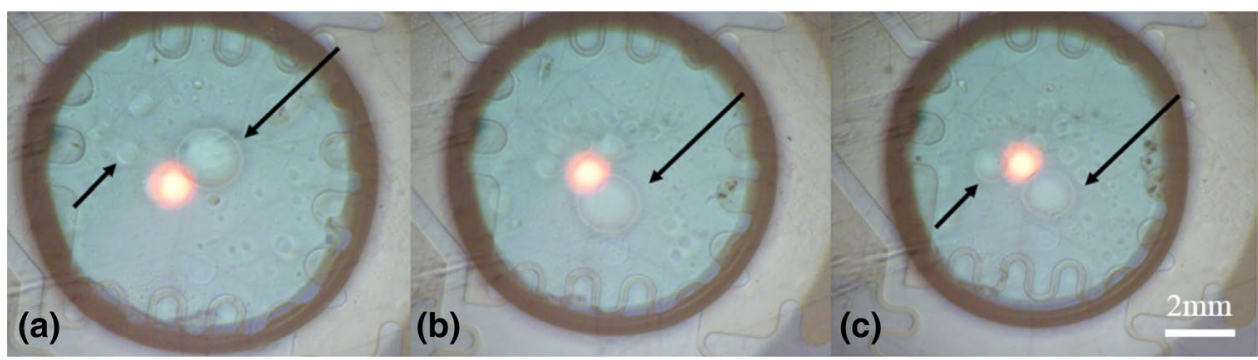

(b)

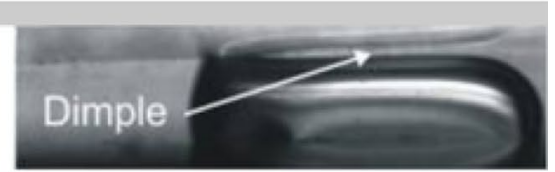

(c)

(a)

(b)

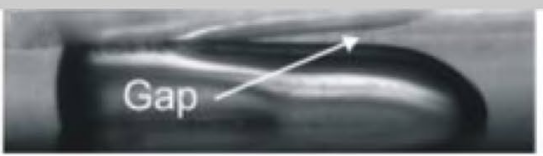

Fig. 11 Side-view photographs showing the formation of oil lenses [16] 


\subsubsection{Voltage polarity switch}

From the previous experiment, oil lenses were observed in the experimental setup used to measure analyte absorption. These lenses could be a local area where the oil films above or under the droplet were thicker, or even the oil film could be ruptured at that point. Earlier studies showed that turning on/off the electrode or changing voltage polarity can change the capacitance between the electrodes with a droplet between them [32]. The change was sometimes repeatable but sometimes semi-random, and it was suspected that the only possible explanation was the thin oil layers. More viscous silicone oil makes the rise in capacitance change of an actuating droplet become slower, and changing actuation voltage levels may cause the droplet to displace oil around it differently. This suggests that changing voltage may also cause a different absorbance measurement.

The polarity switching experiment was designed as follows:

1. 50-V AC (sinusoidal) actuation was applied to the electrodes sequentially to move a 6-ppm sulfate droplet to the detection electrode;

2. The droplet stayed on the detection electrode for $30 \mathrm{~s}$, then the voltage polarity was switched, with the bottom plate detection electrode grounded and the top plate connected to $50 \mathrm{~V}$.

3. After $30 \mathrm{~s}$, the voltage was switched back and the absorbance was measure for another $30 \mathrm{~s}$ before the droplet was moved away. This was repeated for several times. The absorbance over time was plotted. In the first five tests, switching of voltage polarity did not result in a change in absorbance. In the sixth and seventh test groups, the change in voltage polarity caused a significant change in absorbance. In the last three test groups, at the moment the polarity changed, the absorbance increased briefly, probably because the voltage was briefly zero during the volt- age conversion, and the shape of the oil film changed briefly. When the reverse voltage was added, the oil film shape resumed.

Figure 12 shows the first experiment run with 10 measurement groups. It shows that voltage polarity switching does not always affect absorbance, but there is a $25 \%$ chance that it does. This experiment was repeated two more times. In a total of 30 sets of polarity switch tests, 7 groups of tests clearly show that after the voltage polarity changed, the absorbance of the sulfate-MTB-Ba droplet also changed significantly. It is still unclear under what circumstances this may happen.

\subsection{Horizontal light path measurements}

In the previous sections, it was shown that random variation in absorbance measurement was a fatal problem for vertical measurements in DMF chips with a silicone oil medium, and the reason may come from an uneven oil film. To avoid excessive influence of the oil film on the light path, the two optical fibers were moved to the sides of the droplet. There are two advantages to this measurement method: 1) the light path increased from $0.24 \mathrm{~mm}$ to $\sim 1$, and 2) the uneven oil film between the droplet and the top and bottom plates was no longer in the center of the light path. First, horizontal path measurement stability was tested. MTB-Ba sulfate solutions were made as before and a final droplet (90-ppm sulfate) was tested. The droplet was moved in and out of the optical path twenty times, and results showed much more consistent readings than the vertical measurement results, as shown in Fig. 13.

Unlike the vertical measurement method, the droplet was maintained between two electrodes. By turning on individual electrodes, it was easy to adjust the droplet position relative to the optical fibers. The entering and exiting movements of the droplet created spikes in absorbance measurements in the graph, and when the droplet was kept at the detecting position, the

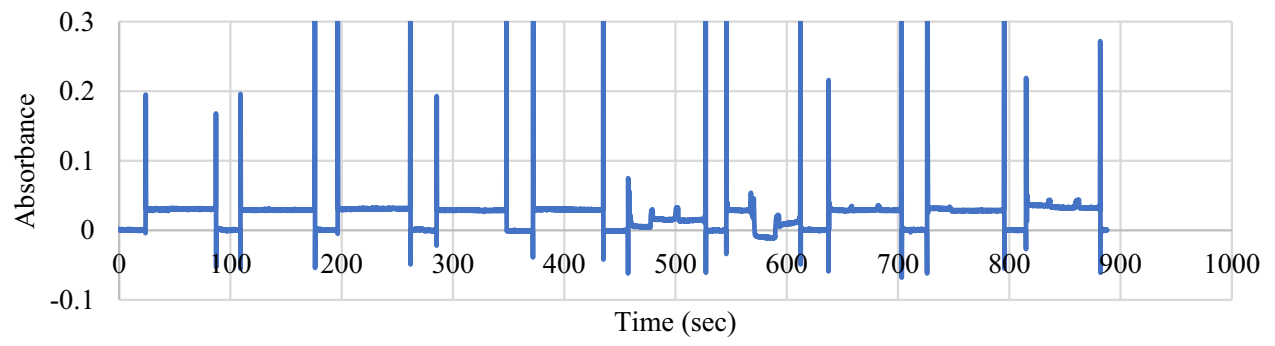

Fig. 12 The absorbance changes with voltage polarity switches applied to a 6-ppm sulfate droplet over the detection electrode. The spikes indicate the droplet moving in and out of position. Between two spikes $(90 \mathrm{~s})$, the polarity switching happens at $30 \mathrm{~s}$ and $60 \mathrm{~s}$, while the droplet did not move. In the first five tests, the voltage polarity switch points were not obvious. In the latter groups of tests, the point of the polarity switch was shown by a significant change in absorbance, which is easy to see in the figure 
absorbance values were stable. The optical fibers were in fixed positions, and the droplet deformed to pass between the ends of the fibers, as shown in Fig. 14. The ends of the optical fibers must be covered by a hydrophobic coating, so the fibers do not penetrate through the droplet. Otherwise, the droplet would be pinned by the hydrophilic fibers, stopping further droplet actuation.

As expected, the absorbance values from the horizontal measurement path were much higher relative to vertical path measurements due to a longer light path, and the absorbance measurements made after sequential droplet movements were more consistent. Standard deviation of the 20 measurements' averaged is 0.00513 , which is at the same level as most vertical measurements, but since the average of these 20 measurements for a sulfate concentration of $90 \mathrm{ppm}$ is much higher (1.119), the CV is $0.459 \%$.

Up to this point, the horizontal on-chip measurement method produced stable absorbance values using premixed solutions. The concentrations of solutions were adjusted, and the mixing ratio for on-chip sample mixing was made to be 1:1. The droplet mixing can be completed in a few seconds and transported to the detection position as soon as the mixing finishes.

The same sulfate standards were used (Table 1). The final droplet was moved to the detection position and measured 12 times. Results are shown in Fig. 15. The averages and standard deviations for each solution's 12 measurements are shown in Table 1. Compared to previous vertical measurement results, the horizontal absorbance value range increased from $0.005-0.2$ to $0.8-2$ due to longer light path length. The $R^{2}$ value improved to 0.96 compared to 0.73 for the vertical measurements, and the standard deviation for the $90 \mathrm{ppm}$ horizontal measurements was less than $0.3 \%$ compared to $36 \%$ for the vertical measurements.

\section{Simulation}

\subsection{Simulation of the vertical light path}

The parameters of an oil lens include its position, size, curvature and thickness. A non-sequential model was built
Fig. 13 Consistent absorbance readings with horizontal light path for MTB-Ba sulfate solutions
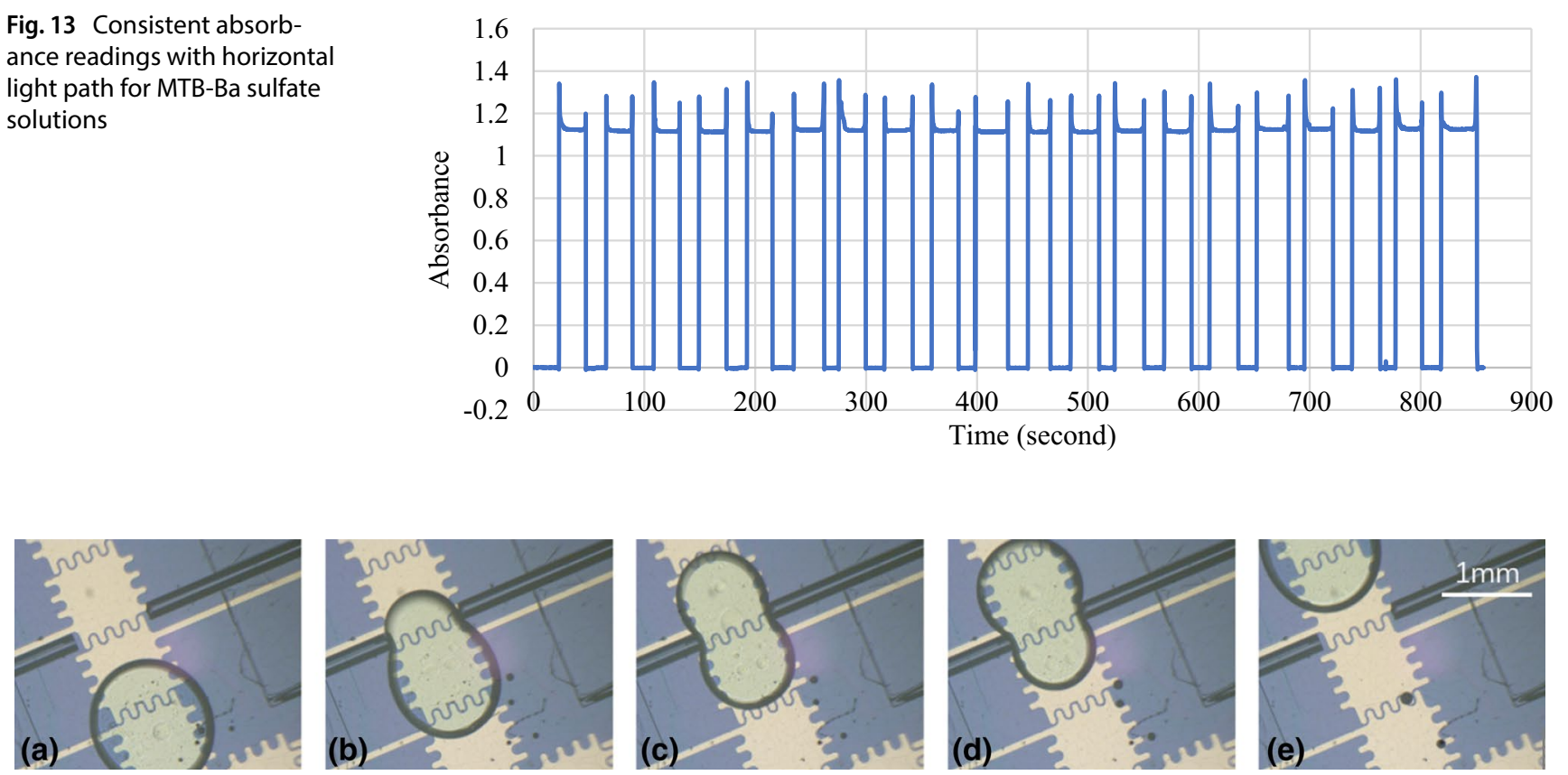

Fig. 14 The movement of an MTB-Ba sulfate solution droplet during a measuring period

Table 1 Statistics of horizontal light path absorbance (averaged over measuring periods) for six concentrations of sulfate

\begin{tabular}{lllllll}
\hline Solution \# & 1 & 2 & 3 & 4 & 5 & 6 \\
Concentration (ppm) & 120 & 90 & 60 & 40 & 12 & 6 \\
Average of 12 measurements & 0.834 & 1.076 & 1.23 & 1.644 & 1.942 & 2.075 \\
SD of 12 measurements & 0.00879 & 0.00386 & 0.0061 & 0.011 & 0.0138 & 0.0554 \\
CV (\%) & 1.05 & 0.359 & 0.496 & 0.67 & 0.71 & 2.67 \\
\hline
\end{tabular}




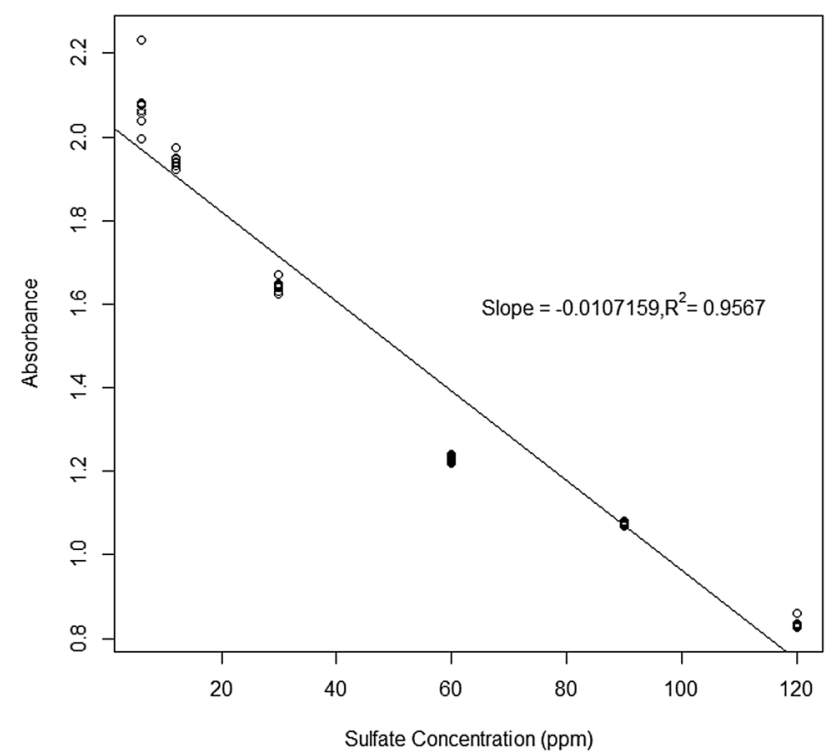

Fig. 15 The 12 measured absorbance (averaged over measuring periods) for six concentrations of sulfate

with Zemax OpticStudio and is shown in Fig. 16. Objects on the vertical light path are shown from left to right in the horizontal direction, and they are bottom plate, water

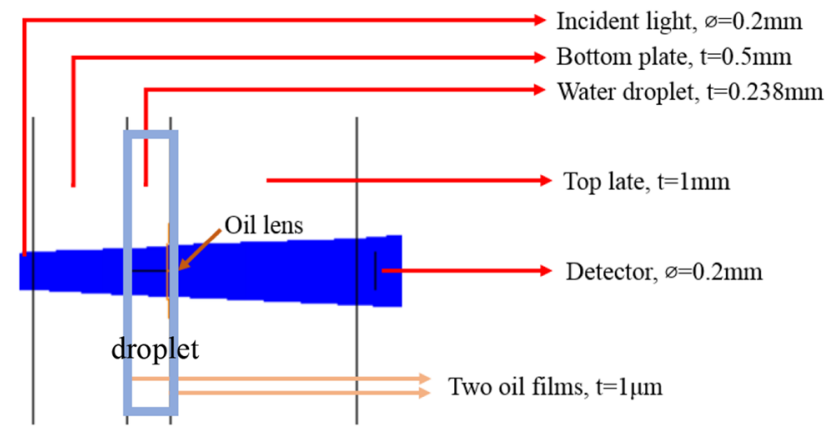

Fig. 16 The schematics of the vertical light path simulation droplet, silicone oil lens, top plate and a circular detector which has the same diameter as the optical fiber. The vertical light path is turned $-90^{\circ}$ in the figure for easier illustration. Simulation and material parameters are listed in Table 2. The light exiting angle was physically measured. The lens is modeled by a spherical cap, and its center is located on the light path. The round cross section is perpendicular to the light path.

Four simulations were designed as follows (Fig. 17): simulation $1 \mathrm{kept}$ the oil lens diameter at $0.6 \mathrm{~mm}$ and varied the oil lens thickness from 0.02 to $0.08 \mathrm{~mm}$; simulation 2 kept the oil lens diameter at $0.2 \mathrm{~mm}$ and varied the oil lens thickness from 0.005 to $0.05 \mathrm{~mm}$; simulation 3 kept the oil lens diameter at $0.6 \mathrm{~mm}$ and thickness at $0.02 \mathrm{~mm}$ and varied the oil lens position (the displacement of the center of the oil lens from the light path) from 0 to $0.3 \mathrm{~mm}$; simulation 4 kept the oil lens diameter at $0.2 \mathrm{~mm}$ and thickness at $0.02 \mathrm{~mm}$ and varied the oil lens position from 0 to $0.2 \mathrm{~mm}$. Because silicone's refractive index is slightly larger than water, the oil lens has a converging effect and will cause an increase in the total power received by the detector. The amount of this increase is shown as a percentage relative to the power received when there was not any oil lens. Figure 18 shows the results.

Several conclusions can be drawn from the results, the most obvious of which is from the largest change in power in simulation 2, where oil lenses with small diameter and large thickness have a greater influence on the optical path. A 0.06-mm-thick oil lens with $0.2 \mathrm{~mm}$ diameter can lead to a $50 \%$ change in the output light intensity. The second largest power change happened in simulation 4, which shows that the position of the oil lens can make a difference of as much as $20 \%$. It is also important to notice that large flat oil lenses do not affect the output light intensity that much. During previous on-chip absorbance measurement experiments, more than one oil lens had been observed, and the overall effect of oil lens size and position can explain the inconsistency of vertical absorbance readings between different measurements.

Table 2 Parameters used in non-sequential simulation

\begin{tabular}{lr}
\hline Parameters & 608 \\
\hline Wavelength (nm) & 10,000 \\
\# Analysis rays & 10 \\
Light source power (lumens) & 8 \\
Optical fiber light exiting angle (degree) & Refractive index \\
\hline Materials & 1.47 \\
\hline Borosilicate glass & 1.38 \\
Silicone oil & 1.33 \\
Water & 1.49 \\
Acrylic & \\
\hline
\end{tabular}




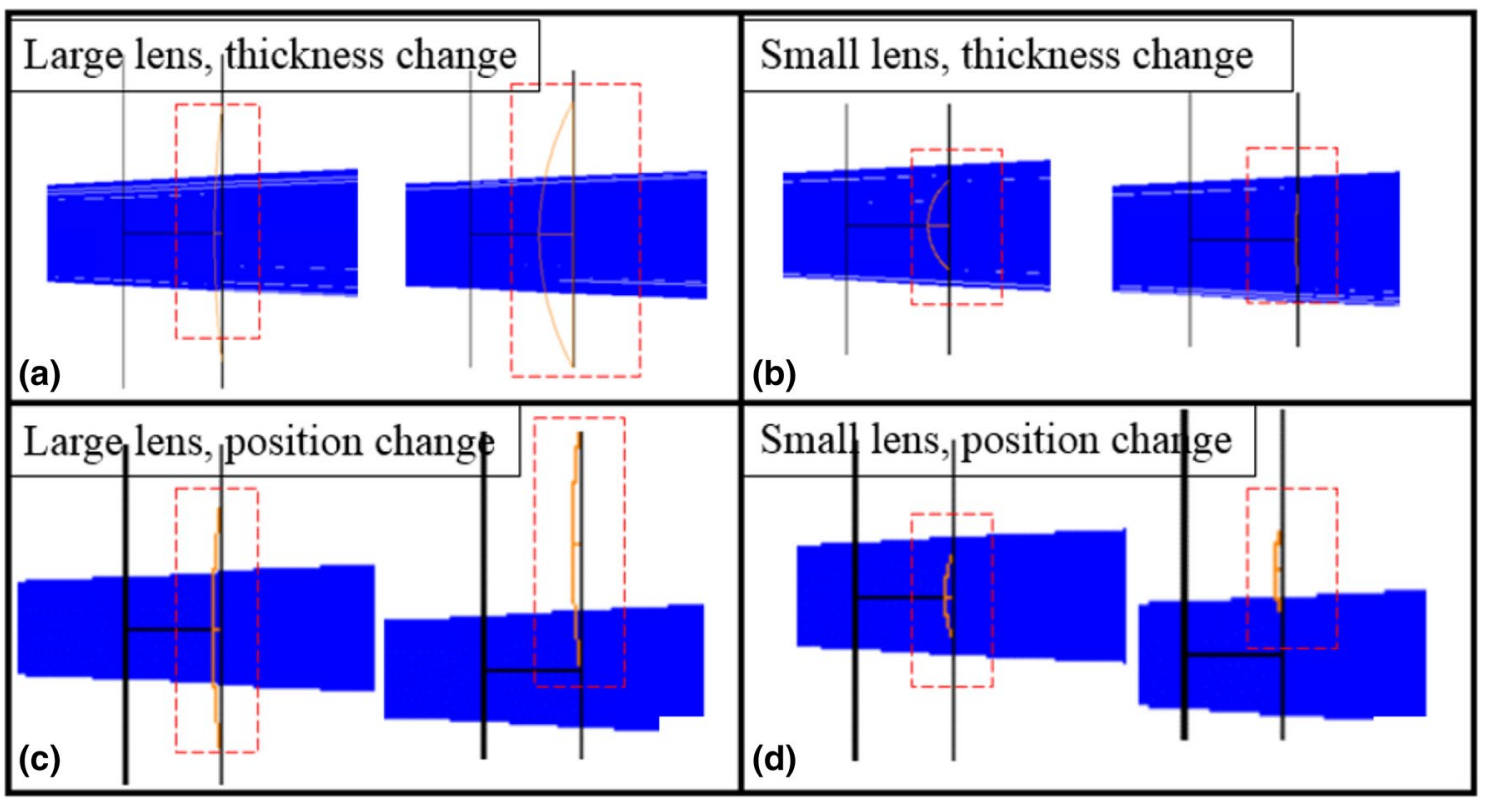

Fig. 17 The relative size and thickness of the oil lens. The orange dotted squares enclose the oil lenses that are also marked with orange lines. The two graphs in each quadrant represent the two extreme situations of the changing variable in the simulation and comparing the two figures reveals how much the thickness or position of the oil lens changes
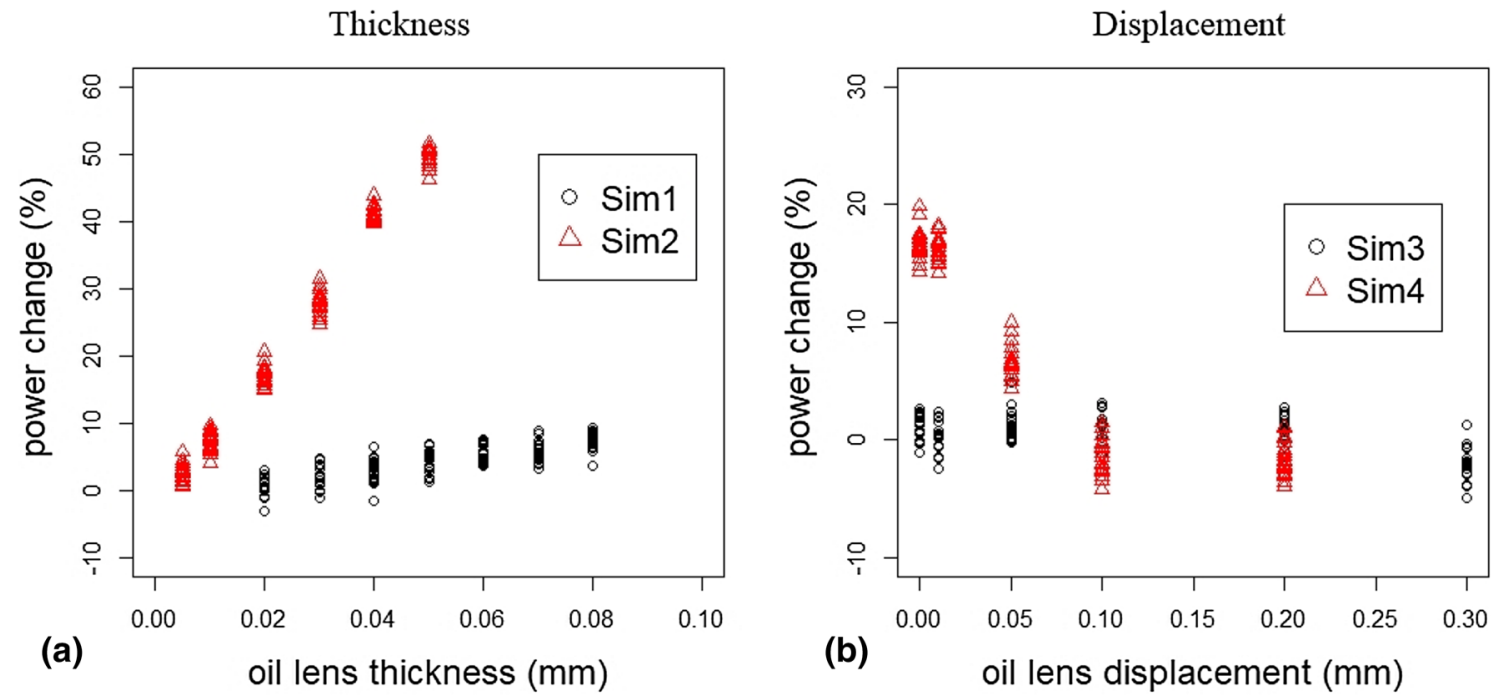

Fig. 18 The change of power (in percentage) received by the photodetector plate for the four simulation conditions in Fig. 17. The replicates are the results of 20 runs with the same set of parameters

In the vertical direction, the light passes through multiple layers of different media and passes through the oil lens, so the light intensity received at the detector is low and easily affected by the position and surface conditions of these materials. If the optical path is in the horizontal direction, these interferences are eliminated.

\subsection{Simulation with horizontal light path}

To test the feasibility of a horizontal light path, two simulations were performed (Figs. 19, 20). For both simulations, the oil lens was placed in the center of the surface over the droplet. Simulation $1 \mathrm{kept}$ the oil lens diameter at $0.6 \mathrm{~mm}$ and varied the thickness from 0.02 to $0.08 \mathrm{~mm}$, 


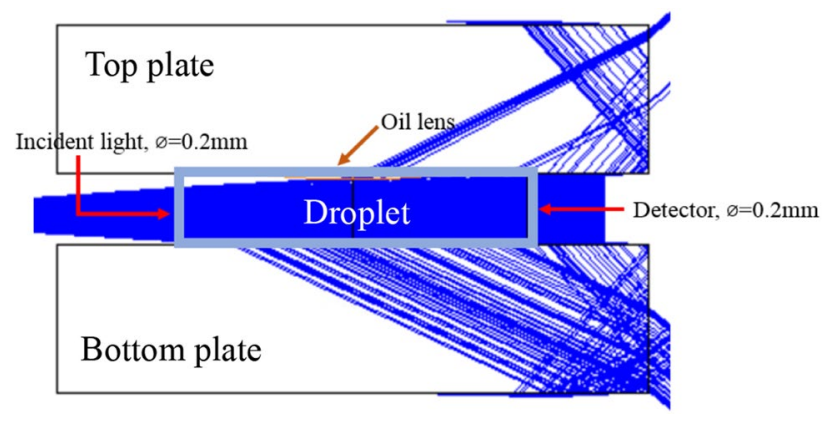

Fig. 19 The cross-sectional view schematics for horizontal light path simulation

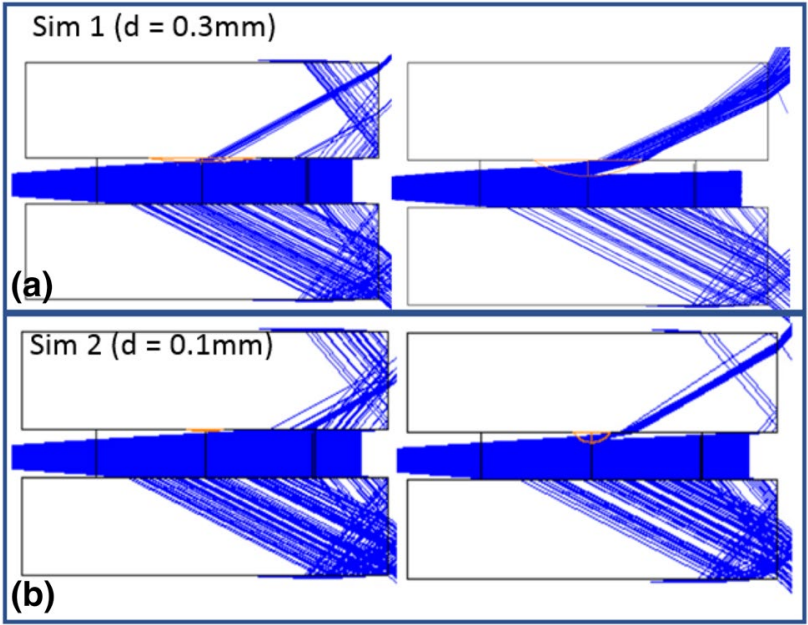

Fig. 20 Horizontal light path simulations. a Horizontal light path simulation 1, b horizontal light path simulation 2. These two sets of graphs show the range of changes in the size and position of the oil lens in these two simulations

while simulation $2 \mathrm{kept}$ the oil lens diameter at $0.2 \mathrm{~mm}$ and varied the thickness from 0.005 to $0.05 \mathrm{~mm}$.

In Fig. 21, the simulations show that the power change caused by oil lens size and thickness was smaller. Therefore, although the appearance and shape of the oil lenses are uncontrollable, these factors do not affect the measurement of the droplet absorbance during a horizontal measurement. Also, a smaller oil lens size seems to have less effect. If most of the oil lenses are small, horizontal measurement provides more stable results than vertical measurements. In addition to this, it was found that on average about $80 \%$ of the incoming light intensity reached the detector with horizontal measurements, while $45 \%$ reached the detector with vertical measurements.

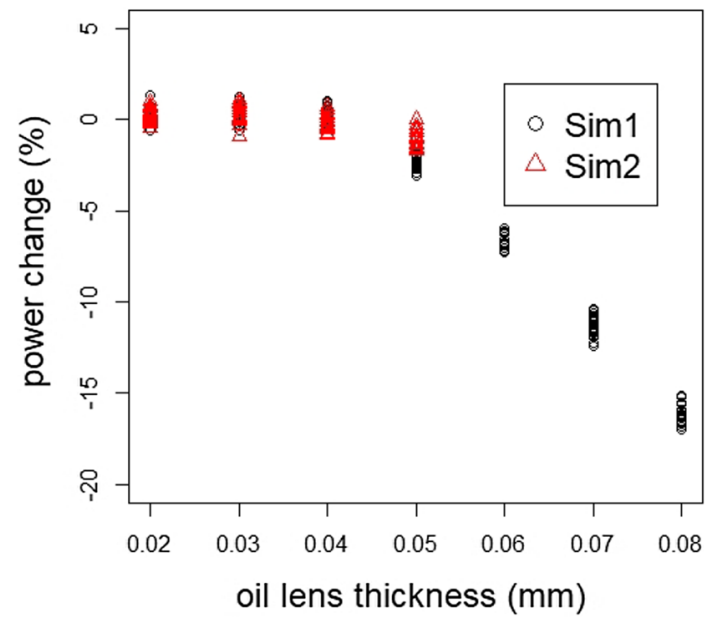

Fig. 21 The change of power (in percentage) received by the photodetector plate. Left: simulation 1; right: simulation 2

\section{Conclusions}

Two methods were studied for selectively measuring the on-chip absorbance of trace sulfate analytes in droplets on a digital microfluidics (DMF) platform operating with a silicon oil medium. Based on the configuration of the chip, two optical fibers were loaded on the chip in either a vertical or a horizontal manner to measure the sample sulfate droplet's absorbance. However, the absorbance measurement using the vertical light path was affected by oil lenses caused by the uneven thickness of the oil film between the droplets and the two plates, so that the same droplet produced different absorbance measurements. After changing the optical path to the horizontal direction, the absorbance is less affected by the oil lens and the measured values are stable.

The horizontal method was part of a detection system of inorganic ions in aerosol and has proved to be successful with sulfate, nitrate and ammonium. Meanwhile, the potential use of the designed system is not limited to atmospheric studies. Applications can extend to testing the quality of drinking water, detection of nitroaromatic explosives or other experiments based on colorimetry.

Acknowledgements This work was supported by the National Science Foundation under Grant NSF AGS-1405362.

\section{Compliance with ethical standards}

Conflict of interest On behalf of all the authors, the corresponding author states that there is no conflict of interest.

Open Access This article is distributed under the terms of the Creative Commons Attribution 4.0 International License (http://creat ivecommons.org/licenses/by/4.0/), which permits unrestricted use, 
distribution, and reproduction in any medium, provided you give appropriate credit to the original author(s) and the source, provide a link to the Creative Commons license, and indicate if changes were made.

\section{References}

1. Abrantes M, Magone MT, Boyd LF, Schuck P (2001) Adaptation of a surface plasmon resonance biosensor with microfluidics for use with small sample volumes and long contact times. Anal Chem 73(13):2828

2. Adamski JM, Villard SP (1975) Application of the methylthymol blue sulfate method to water and wastewater analysis. Anal Chem 47(7):1191-1194

3. Au SH, Shih SCC, Wheeler AR (2011) Integrated microbioreactor for culture and analysis of bacteria, algae and yeast. Biomed Microdev 13(1):41-50

4. Bêche B, Pelletier N, Gaviot E, Zyss J (2004) Single-mode TE00TM00 optical waveguides on SU-8 polymer. Opt Commun 230(1-3):91-94

5. Cai Z, Qiu W, Shao G, Wang W (2013) A new fabrication method for all-PDMS waveguides. Sens Actuators A 204:44-47

6. Carter $P$ (1971) Spectrophotometric determination of serum iron at the submicrogram level with a new reagent (ferrozine). Anal Biochem 40(2):450-458

7. Ceyssens F, Witters D, Van Grimbergen T, Knez K, Lammertyn J, Puers R (2013) Integrating optical waveguides in electrowettingon-dielectric digital microfluidic chips. Sens Actuators B Chem 181:166-171

8. Chang-Yen DA, Eich RK, Gale BK (2005) A monolithic PDMS waveguide system fabricated using soft-lithography techniques. J Lightwave Technol 23(6):2088

9. Chapman DV, World Health Organization (1996) Water quality assessments: a guide to the use of biota, sediments and water in environmental monitoring, 2nd edn. E and FN Spon, London

10. Chen L, Fair RB (2015) Digital microfluidics chip with integrated intra-droplet magnetic bead manipulation. Microfluid Nanofluid 19(6):1335-1348

11. Choi K, Mudrik JM, Wheeler AR (2015) A guiding light: spectroscopy on digital microfluidic devices using in-plane optical fibre waveguides. Anal Bioanal Chem 407:7467-7475

12. Fair RB, Khlystov A, Srinivasan V, Pamula VK, Weaver KN (2004) Integrated chemical/biochemical sample collection, pre-concentration, and analysis on a digital microfluidic lab-on-a-chip platform. In: Lab-on-a-chip: platforms, devices, and applications, vol 5591, pp 113-125. International Society for Optics and Photonics

13. Hong J, Kim YK, Won DJ, Kim J, Lee SJ (2015) Three-dimensional digital microfluidic manipulation of droplets in oil medium. Sci Rep 5:10685. https://doi.org/10.1038/srep10685

14. Huang $S$ (2018) Digital microfluidics for the detection of inorganic ions in aerosols. Ph.D. Dissertation, Duke University (unpublished)

15. Jung DG, Jung D, Kong SH (2017) A lab-on-a-chip-based noninvasive optical sensor for measuring glucose in saliva. Sensors 17(11):2607
16. Kleinert J, Srinivasan V, Rival A, Delattre C, Velev OD, Pamula VK (2015) The dynamics and stability of lubricating oil films during droplet transport by electrowetting in microfluidic devices. Biomicrofluidics 9(3):034104

17. Macounova K, Cabrera CR, Yager P (2001) Concentration and separation of proteins in microfluidic channels on the basis of transverse IEF. Anal Chem 73(7):1627

18. Madsen BC, Murphy RJ (1981) Flow injection and photometric determination of sulfate in rainwater with methylthymol blue. Anal Chem 53(12):1924-1926

19. Marczenko $Z$ (1986) Separation and spectrophotometric determination of elements. OSTI ID: 6723640

20. McSwain MR, Watrous RJ, Douglass JE (1974) Improved methylthymol blue procedure for automated sulfate determinations. Anal Chem 46(9):1329-1331

21. Miranda KM, Espey MG, Wink DA (2001) A rapid, simple spectrophotometric method for simultaneous detection of nitrate and nitrite. Nitric Oxide 5(1):62-71

22. Paik P, Pamula VK, Chakrabarty K (2004) Thermal effects on droplet transport in digital microfluidics with applications to chip cooling. In: Proceedings of the IEEE, pp 649-654

23. Perkampus HH (2013) UV-VIS spectroscopy and its applications. Springer, Berlin

24. Prieto P, Pineda M, Aguilar M (1999) Spectrophotometric quantitation of antioxidant capacity through the formation of a phosphomolybdenum complex: specific application to the determination of vitamin E. Anal Biochem 269(2):337-341

25. Pollack MG, Fair RB, Shenderov AD (2000) Electrowetting-based actuation of liquid droplets for microfluidic applications. Appl Phys Lett 77:1725-1726

26. Schmid FX (2001) Biological macromolecules: UV-visible spectrophotometry. https://doi.org/10.1038/npg.els.0003142

27. Srinivasan V, Pamula VK, Fair RB (2004) Droplet-based microfluidic lab-on-a-chip for glucose detection. Anal Chim Acta 507(1):145-150

28. Swinehart DF (1962) The Beer-lambert law. J Chem Educ 39(7):333

29. Tan Y-C, Fisher JS, Lee Al, Cristini V, Lee AP (2004) Design of microfluidic channel geometries for the control of droplet volume, chemical concentration, and sorting. Lab Chip 4:292-298

30. Wang H, Chen L, Sun L (2017) Digital microfluidics: a promising technique for biochemical applications. Front Mech Eng 12(4):510-525

31. Wijethunga PAL, Nanayakkara YS, Kunchala P, Armstrong DW, Moon H (2011) On-chip drop-to-drop liquid microextraction coupled with real-time concentration monitoring technique. Anal Chem 83:1658-1664

32. Woo R (2001) Capacitance and microfluidics. Independent Study Project. Duke University (unpublished)

Publisher's Note Springer Nature remains neutral with regard to jurisdictional claims in published maps and institutional affiliations. 